\title{
Aplicación de los diagnósticos de enfermería en el ámbito de la salud laboral. Ejemplos de prescripción enfermera
}

\author{
Application of nursing diagnoses in the field of occupational health. Examples \\ of nurse prescribing
}

Javier González Caballero

Instituto Nacional de la Seguridad Social. Bilbao (Vizcaya). España

Recibo: 24-06-09

Aceptado: 26-10-10

\section{Correspondencia:}

Javier González Caballero

Instituto Nacional de la Seguridad Social

Gran Vía, 89- $1^{\circ}$

48011 Bilbao. España

Tfno: 944284500

e-mail: javiergc_48@yahoo.es

Resumen

En la VIII Conferencia de la North American Nursing Diagnosis Association (NANDA) de 1988 se presenta a la Organización Mundial de la Salud (OMS) una versión específica de una taxonomía diagnóstica enfermera con el fin de ser incluida en la revisión de la Clasificación Internacional de Enfermedades (CIE-10) como un sistema idóneo para clasificar la práctica enfermera. A partir de las recomendaciones de la propia OMS se elaboran diversos documentos relacionados con la integración de los diagnósticos e intervenciones de enfermería en la práctica profesional. Estas actuaciones permiten de una parte una difusión de un lenguaje común y propio a la profesión, y de otra una sistematización del trabajo. De esta forma se incorporan distintos diagnósticos de enfermería aplicados en el ámbito de la especialidad de Enfermería del Trabajo, determinando en cada caso las intervenciones que un profesional podría realizar acorde con sus competencias y de esta forma permitir un desarrollo de la profesión basado en la evidencia científica.

Med Segur Trab (Internet) 2010; 56 (221): 328-346

Palabras clave: enfermería del trabajo, prescripción enfermera.

\begin{abstract}
In the VIII Conference of the North American Nursing Diagnosis Association (NANDA) in 1988 is presented to the World Health Organization (WHO), a specific version of a diagnostic taxonomy nurse in order to be included in the revision of the International Classification of Diseases (ICD-10) as an appropriate system for classifying nursing practice. Based on the recommendations of the WHO itself are produced various documents relating to the integration of nursing diagnoses and interventions in professional practice. These actions allow a part of a spread of a common language and the profession itself, and other systematic work. This will incorporate the different nursing diagnoses used in the nursing specialty of Labour, in each case identifying the interventions that could make a career commensurate with their skills and enabling the development of a profession based on scientific evidence.
\end{abstract}

Med Segur Trab (Internet) 2010; 56 (221): 328-346

Key words: occupational nurse, nurse prescribing. 


\section{INTRODUCCIÓN}

Desde hace varias décadas en la literatura enfermera se discute la necesidad de unificar o estandarizar el lenguaje enfermero ${ }^{1}$. En esta línea de trabajo son varios los autores de reconocida trayectoria profesional: Maas 1, McCormick 2,3, Zielstroff 4,5, McCloskey y Bulechek ${ }^{6}$, Moss ${ }^{7}$, Aquilino y Keenan ${ }^{8}$, Dochterman y Jones 9 ...

Cuando se utiliza un lenguaje normalizado para documentar la práctica profesional, se puede comparar y evaluar la efectividad de los cuidados suministrados en múltiples situaciones por profesionales de la enfermería. El uso del lenguaje normalizado hace visible la esencia de los cuidados enfermeros y ayuda en la mejora de la práctica cotidiana. Su desarrollo y utilización sirve de apoyo para avanzar en el conocimiento de los cuidados facilitando la aplicación clínica de las intervenciones de enfermería ${ }^{10}$.

Un lenguaje enfermero estandarizado tiene varios propósitos:

- Proporciona una herramienta a los profesionales de la enfermería para comunicar lo que realizan entre ellos mismos, en colaboración con otros profesionales sanitarios y con la población destinataria de los cuidados.

- Permite la recogida y el análisis de la información documentando la contribución enfermera al cuidado del paciente.

- Facilita la evaluación y la mejora de los cuidados enfermeros.

- Favorece el desarrollo del conocimiento enfermero.

- Permite el desarrollo de sistemas de información clínica y de registro de datos.

- Proporciona información sobre la elaboración de políticas organizativas respecto a la atención sanitaria y enfermera.

- Permite la universalización de la nomenclatura diagnóstica y de las intervenciones enfermeras.

La American Nurses Association (ANA) elabora tres tipos de lenguajes estandarizados:

- Los Diagnósticos Enfermeros desarrollados por la NANDA (North American Nursing Diagnosis Association) International.

- La Clasificación de Intervenciones de Enfermería (NIC).

- La Clasificación de Resultados de Enfermería (NOC).

Las relaciones entre estas clasificaciones son el principal paso para facilitar el uso de estos lenguajes en la práctica diaria, en los estudios de pregrado y posgrado, en el ámbito de la investigación y en la formación continuada.

\section{OBJETIVOS}

La especialidad de Enfermería del Trabajo cuenta con una larga trayectoria en el ámbito de la atención especializada por su formación específica. La capacitación profesional para desarrollar sus competencias viene refrendada por un soporte normativo constituido básicamente por la publicación de la siguiente legislación:

- La Ley 31/1995 de 8 de noviembre, de Prevención de riesgos laborales, y su modificación posterior en la Ley 54/2003, de 12 de diciembre, de reforma del marco normativo de la Prevención de riesgos laborales;

- el Real Decreto 39/1997 de 17 de enero, por el que se aprueba el Reglamento de los servicios de prevención, y su modificación recogida en el Real Decreto $337 / 2010$ de 17 de enero;

- el Real Decreto 1231/2001, de 8 de noviembre, de Ordenación de la actividad profesional de enfermería; 
- la Ley 16/2003, de 28 de mayo, de Cohesión y calidad del Sistema Nacional de Salud;

- la Ley 44/2003 de 21 de noviembre de Ordenación de las profesiones sanitarias; el Real Decreto 450/2005, de 22 de abril, sobre especialidades de Enfermería donde queda recogida en el artículo 2 la especialidad de Enfermería del Trabajo,

- y por último, la Orden SAS/1348/2009 de 6 de mayo por la que se aprueba y publica el programa formativo de la especialidad de Enfermería del Trabajo marcan un momento relevante en el desarrollo de las competencias de esta disciplina enfermera.

La Constitución de la Organización Mundial de la Salud (OMS), la Declaración de Alma-Ata sobre Atención Primaria de Salud, la Estrategia Mundial OMS de la Salud Ocupacional para Todos, el Convenio sobre seguridad y salud de los trabajadores número 155 y su Recomendación de acompañamiento número 164 de la Organización Internacional del Trabajo (OIT), el Convenio sobre los servicios de salud en el trabajo número 161 de la OIT y su Recomendación de acompañamiento número 171, y la Directiva Marco de la Comunidad Europea 1989/391/CEE, señalan de una parte el derecho fundamental de cada trabajador a recibir los estándares más altos de cuidados de salud en su ámbito de trabajo, y orientan a las oportunas modificaciones legislativas en la práctica de la salud laboral.

Considerando el marco normativo descrito, la experiencia específica en cada ámbito de actuación y los conocimientos y habilidades necesarios para el ejercicio profesional, se establecen los siguientes objetivos:

- Identificar las competencias profesionales de la Enfermería del Trabajo.

- Establecer los diagnósticos enfermeros de aplicación en su ámbito de competencias.

- Especificar las intervenciones, actividades y resultados que se pretenden una vez establecidos los diagnósticos de Enfermería aplicando la terminología NANDA, NIC y NOC.

- Fomentar e interrelacionar la terminología NANDA, NIC y NOC en el marco de la salud laboral.

\section{DIAGNÓSTICOS DE ENFERMERÍA. MARCO TEÓRICO}

\section{NANDA Internacional}

El uso del lenguaje enfermero estandarizado comenzó a gestarse en 1973 con el desarrollo de la clasificación diagnóstica de la NANDA. Un diagnóstico enfermero es un juicio clínico sobre las respuestas individuales, familiares o de la comunidad a problemas de salud o procesos vitales reales o potenciales. Un diagnóstico enfermero proporciona la base para elegir las intervenciones enfermeras que pretenden conseguir los resultados de los que el profesional enfermero es responsable ${ }^{11}$.

\section{NIC. Nursing Interventions Classification}

La investigación para desarrollar un vocabulario y una clasificación de intervenciones enfermeras surge en 1987 con la formación de un equipo de investigación conducido por McCloskey y Bulechek en la Universidad de Iowa, USA. El equipo desarrolló la Clasificación de Intervenciones de Enfermería (NIC), una clasificación global y estandarizada de las intervenciones que realizan las enfermeras publicada por primera vez en 1992. A diferencia de los diagnósticos enfermeros donde el centro de interés es el paciente, en las intervenciones de enfermería el interés se traslada a la conducta enfermera; en definitiva 
todo aquello que los profesionales de la enfermería realizan para ayudar al paciente a avanzar hacia un resultado deseado.

Una intervención se define como cualquier tratamiento basado en el conocimiento y juicio clínico que realiza un profesional de enfermería para favorecer los resultados esperados del paciente/cliente. Las intervenciones incluyen todas las acciones ejecutadas por las enfermeras, tanto los cuidados directos como los cuidados indirectos dirigidos a los individuos, las familias o la comunidad, ya sean tratamientos iniciados por las enfermeras, por los médicos o por otros profesionales ${ }^{12}$. Las intervenciones estandarizadas del NIC nos ayudan a documentar los cuidados. Estos pueden individualizarse a través de la elección de las actividades. A partir de una lista de aproximadamente entre 10 y 30 actividades por intervención, el profesional de la enfermería elige las más adecuadas para un individuo o familiar concreto.

\section{NOC. Nursing Outcomes Classification}

En 1991 se creó un equipo de investigación dirigido por M. Johnson y M. Maas en la Universidad de Iowa (USA) para desarrollar una clasificación de los resultados de los pacientes que se correlacionan con los cuidados enfermeros. El trabajo del equipo de investigación dio como resultado la Clasificación de Resultados de Enfermería (NOC), una clasificación estandarizada de los resultados que se espera obtener con el paciente y que pueden utilizarse para evaluar los resultados de las intervenciones enfermeras (NIC). Fue publicado por primera vez en 1997.

Se define un resultado como un estado, una conducta o percepción individual, familiar o comunitaria que se mide a lo largo de un continuo en respuesta a una intervención enfermera ${ }^{13}$. Es conocido que numerosas variables junto con la intervención influyen en los resultados finales con el paciente. Estas variables pueden ser: el proceso utilizado en el suministro del cuidado, las acciones de otros profesionales sanitarios, el perfil de la organización, o aspectos del entorno que influyen en la selección y el suministro de intervenciones según las características del paciente (estado físico, emocional y circunstancias vitales y profesionales).

Por tanto la relación de los tres lenguajes proporciona ayuda a los profesionales clínicos para elegir los resultados (NOC) y las intervenciones (NIC) más adecuadas para sus pacientes en relación con los diagnósticos enfermeros.

\section{PRESCRIPCIÓN ENFERMERA. MARCO CONCEPTUAL}

El Gabinete de Estudios del Consejo General de Enfermería sugiere las siguientes definiciones de los términos prescribir y recetar ${ }^{14}$.

Prescribir: emitir un criterio profesional sanitario dirigido al paciente por el que se propone la realización de una acción en virtud de un juicio clínico, terapéutico o de cuidados.

Recetar: cumplimentar un formulario que contiene una medicación o el objeto de la prescripción que el profesional sanitario indica a un paciente acompañándose de instrucciones genéricas sobre su uso, y que normalmente se expide para ser presentado en una farmacia para su adquisición.

En este marco conceptual resulta de interés definir producto sanitario tal y como se recoge en la Ley de Garantías y uso racional de los medicamentos ${ }^{15}$ en su artículo 8: cualquier instrumento, dispositivo, equipo, material u otro artículo, utilizado solo o en combinación, incluidos los programas informáticos que intervengan en su buen funcionamiento, destinados por el fabricante a ser utilizados en seres humanos con fines, entre otros, de diagnóstico, prevención, control, tratamiento o alivio de una enfermedad. 
Posteriormente, a través de la Ley 28/2009, de 30 de diciembre, de modificación de la Ley 29/2006, de 26 de julio, de Garantías y uso racional de los medicamentos y productos sanitarios señala que los enfermeros de forma autónoma podrán indicar, usar y autorizar la dispensación de todos aquellos medicamentos no sujetos a prescripción médica y también los productos sanitarios mediante la correspondiente orden de dispensación. El Gobierno regulará la indicación, uso y autorización de dispensación de determinados medicamentos sujetos a prescripción médica por los enfermeros en el marco de los principios de la atención integral de salud y para la continuidad asistencial en el ámbito de los cuidados tanto generales como especializados. Y todo ello mediante la aplicación de protocolos y guías de práctica clínica y asistencia de elaboración conjunta, acordados con las organizaciones colegiales de médicos y enfermeros y validados por la Agencia de Calidad del Sistema Nacional de Salud. El Ministerio de Sanidad y Política Social con la participación de las organizaciones colegiales referidas anteriormente, acreditará con efectos en todo el Estado, a los enfermeros para las actuaciones previstas en este artículo.

Bulecheck y McCloskey (1992) definen prescripción enfermera como cualquier cuidado directo que la enfermera realiza en beneficio del cliente. El cuidado directo incluye:

- Los tratamientos iniciados por la enfermera,

- los tratamientos iniciados en función del diagnóstico médico y

- la realización de actividades diarias esenciales para el paciente.

La prescripción enfermera ${ }^{16,17}$ debe entenderse como la capacidad de los enfermeros en el marco de sus competencias y atribuciones para contribuir de manera más eficaz en:

- La gestión de los problemas de salud o de enfermedad de los ciudadanos.

- La gestión de las prestaciones sanitarias, asistenciales y de cuidados.

- La optimización de los recursos.

- El desarrollo del rol autónomo de la enfermería.

Bigbee ${ }^{18}$ señala que la prescripción enfermera no ha de delimitarse o circunscribirse a los fármacos sino también debe incluir el mejor régimen terapéutico posible. Prescribir es una responsabilidad profesional que ha de ser asumida y aplicada. Es consecuencia de la práctica profesional para la que el enfermero/a se encuentra habilitado y de la cual forma parte activa.

De esta manera se puede entender por prescripción enfermera como la capacidad del enfermero/a, en el marco de la dirección, evaluación y prestación de los cuidados de enfermería, de seleccionar, guiado por el criterio profesional, diferentes materiales, productos, dispositivos y medicación encaminados a satisfacer las necesidades de salud del usuario y la población, apoyados por el juicio clínico enfermero y administrados en forma de cuidados.

\section{ENFERMERÍA DEL TRABAJO. MARCO DE COMPETENCIAS}

\section{Reseña normativa cronológica}

La Ley 31/1995, de 8 de noviembre, de Prevención de Riesgos Laborales ${ }^{19}$ (LPRL), y su desarrollo posterior a través del Real Decreto 39/1997, de 17 de enero, por el que se aprueba el Reglamento de los Servicios de Prevención ${ }^{20}$ (RSP), constituyen la legislación específica que regula las actuaciones de la Enfermería del Trabajo en su actividad laboral. Tanto la LPRL como el RSP entienden la prevención y la protección de la salud del trabajador con una mentalidad proactiva ${ }^{21}$. De esta forma se contempla la prevención como una actividad integrada que ha de contar con asesoramiento y asistencia técnica multidisciplinar desde los servicios de prevención con la aportación de las distintas 
disciplinas como Seguridad, Higiene Industrial, Medicina del Trabajo, Ergonomía y Psicosociología aplicada. A partir de la publicación del RSP, la Enfermería del Trabajo se define como una especialidad con entidad propia dentro de la disciplina de la Medicina del Trabajo, en base a sus competencias profesionales.

Posteriormente, la Ley 44/2003, de 21 de noviembre, de Ordenación de las Profesiones Sanitarias ${ }^{22}$, en su artículo 2 del Título Preliminar recoge aspectos relativos a las profesiones sanitarias tituladas en cuanto a conocimientos, habilidades y actitudes propias de la atención de salud. Señalando a su vez aspectos profesionales referidos a las funciones de investigación, docentes, de gestión clínica, de prevención, información y educación sanitaria.

Específicamente en el artículo 7 de la citada Ley dirigido a los diplomados sanitarios, reseña que les corresponde la prestación personal de los cuidados o los servicios propios de su competencia profesional en las distintas fases del proceso de atención de salud, sin menoscabo de la competencia, responsabilidad y autonomía propias de los distintos profesionales que intervienen en el proceso. En el mismo artículo indica que a los enfermeros se les confieren las funciones de dirección, evaluación y prestación de cuidados de enfermería orientados a la promoción, mantenimiento y recuperación de la salud, así como a la prevención de las enfermedades y discapacidades.

A través del Real Decreto 450/2005, de 22 de abril, sobre especialidades de Enfermería ${ }^{23}$ donde reconoce y regula reglamentariamente las especialidades de Enfermería, entre ellas la de Enfermería del Trabajo ${ }^{24,25}$ y concibe el cometido profesional de esta disciplina como una profesión con autonomía, funciones y responsabilidades propias.

Finalmente la Orden SAS/1348/2009 de 6 de mayo por la que se aprueba y publica el Programa formativo de la especialidad de Enfermería del Trabajo establece un punto de inflexión en esta disciplina enfermera señalando aspectos muy relevantes. La Comisión Nacional de la especialidad de Enfermería del Trabajo elabora el primer programa formativo en el marco de las previsiones contenidas en la Ley 31/1995 de 8 de noviembre de Prevención de riesgos laborales, donde define esta disciplina enfermera, establece los ámbitos de actuación profesional, las funciones y las competencias tanto básicas de carácter transversal como específicas en las distintas áreas: preventiva, asistencial, legal y pericial, gestión, docencia e investigación.

La formación en la especialidad de enfermería del trabajo tiene como objetivo formar a profesionales que puedan realizar las actividades propias de su especialidad, dirigidas a prevenir, promover, proteger y restaurar la salud de la población trabajadora con un enfoque laboral y comunitario, integrado e integral, con una base científica y un conocimiento empírico.

\section{Competencias básicas de carácter transversal}

- Resolver problemas en entornos nuevos o poco conocidos dentro de contextos más amplios relacionados con la enfermería del trabajo.

- Transmitir información, ideas, problemas y soluciones en el ámbito de la especialidad.

- Integrar conocimientos y enfrentarse a situaciones complejas, emitiendo juicios que tengan en cuenta los principios deontológicos y éticos de la profesión, así como las circunstancias sociales del medio en el que se ejerce.

- Habilidad de aprendizaje que le permita continuar estudiando de un modo que habrá de ser en gran medida autodirigido o autónomo.

- Tomar decisiones basadas en principios éticos, conocimientos técnicos y evidencia científica.

- Aplicar sus conocimientos a su trabajo en el equipo multidisciplinar de los servicios de prevención o en los que desarrolle su actividad. 


\section{Competencias específicas}

Las competencias profesionales que deben adquirir los enfermeros especialistas en enfermería del trabajo, se pueden agrupar en cinco grandes áreas: preventiva, asistencial, legal y pericial, de gestión y docente e investigadora.

\section{Área preventiva}

- Incrementar el nivel de salud de la población trabajadora, mediante acciones encaminadas a la promoción de la salud, prevención de riesgos derivados del trabajo, protección de la salud y formación de los trabajadores.

- Detectar precozmente los factores de riesgo derivados del trabajo, así como cualquier alteración que pueda presentarse en los trabajadores tanto sanos como enfermos, llevando a cabo las actividades necesarias para el control y seguimiento de los mismos, profundizando en la población especialmente sensible.

- Promover la participación activa del trabajador en medidas de protección frente a los riesgos a los que están expuestos en su trabajo, fomentando la responsabilidad laboral en materia de salud y seguridad, promoviendo la participación de la población trabajadora en planes de emergencia.

- Colaborar, participar y, en su caso, dirigir las actividades de planificación y dirección relativas a la implantación de los planes de emergencia.

- Realizar campañas de promoción de la salud y fomentar una cultura de salud en el ámbito de su competencia.

- Asesorar a los órganos de dirección de las empresas e instituciones sobre los riesgos y la repercusión de las condiciones de trabajo sobre la salud de los trabajadores.

- Interactuar con los distintos agentes sociales: organizaciones sindicales y sociedades científicas para el desarrollo de más recursos y nuevas iniciativas para la mejora de la salud y la seguridad de los trabajadores.

\section{Área asistencial}

- Identificar problemas de salud laboral reales o potenciales.

- Proporcionar una atención integral a los trabajadores, ayudándoles en la recuperación de la salud y en la reincorporación al trabajo, ante todo tipo de proceso con especial incidencia en las alteraciones de origen laboral.

- Manejar correctamente medicamentos y productos sanitarios relacionados con los cuidados de salud laboral, con el nivel de competencia fijado por la legislación vigente.

- Proponer incentivar la continuidad de los tratamientos y cuidados proporcionados a la población trabajadora, tanto dentro como fuera de su jornada laboral, estableciendo los canales de comunicación adecuados, coordinado si es necesario con otros profesionales sanitarios.

- Colaborar en el equipo multidisciplinar y, en su caso, evaluar y documentar en el ámbito de sus competencias, la aptitud de los trabajadores para el desempeño de su trabajo, realizando los informes oportunos.

- Prestar atención de urgencia y emergencia en el centro de trabajo donde realice su actividad.

- Realizar los procedimientos de cirugía menor relacionados con los cuidados propios del ámbito de la salud laboral.

\section{Área legal y pericial}

- Valorar pericialmente, en el ámbito competencial de la profesión enfermera, las consecuencias que tiene la patología derivada del entorno laboral para la salud humana, en particular en caso de las incapacidades. 
- Valorar pericialmente, en el ámbito competencial de la profesión enfermera, las consecuencias que tiene la patología común que pudiera colisionar con la actividad laboral.

- Detectar y valorar en el ámbito competencial de la profesión enfermera, los estados de salud que sean susceptibles de cambio de puesto de trabajo.

- Valorar pericialmente, en el ámbito competencial de la profesión enfermera, las actuaciones sanitarias en el ámbito laboral.

- Asesorar y orientar en la gestión y tramitación de los expedientes de incapacidad ante las entidades y los organismos competentes.

\section{Área de gestión}

- Realizar estudios, desarrollar protocolos, evaluar programas y guías de actuación para la práctica de la enfermería del trabajo.

- Gestionar cuidados dirigidos a elevar el nivel de calidad y que permitan valorar el coste/efectividad de los mismos.

- Trabajar y colaborar activamente en el equipo multidisciplinar de los servicios de prevención ocupando su puesto de enfermero/a especialista.

- Gestionar y organizar los servicios de prevención de riesgos laborales en sus distintas modalidades y ámbitos.

- Coordinar sus actuaciones con el Sistema Nacional de Salud.

- Gestionar un sistema de registro apropiado y accesible que asegure la confidencialidad de los datos de acuerdo con lo previsto en la legislación vigente.

\section{Área de docencia e investigación}

- Realizar comunicaciones científicas y de divulgación.

- Informar y formar a la población trabajadora, planificando, ejecutando y evaluando programas educativos según las necesidades detectadas, de forma individualizada o en grupos.

- Proponer e intervenir en proyectos de investigación, encaminados a mejorar continuamente la actividad profesional y con ello elevar la salud de la población trabajadora.

- Utilizar y mejorar instrumentos que permitan medir los resultados de las intervenciones enfermeras.

\section{METODOLOGÍA}

Considerando el marco legislativo precedente y el rol de la Enfermería del Trabajo se han definido cada una las competencias que constituyen la actividad profesional como parte integrante de los servicios de prevención de riesgos laborales. Las competencias se agrupan en distintos ámbitos de actuación donde algunas son estrictamente sanitarias y otras se realizan conjuntamente y de forma coordinada con otros profesionales del servicio de prevención. A su vez el análisis de las situaciones más prevalentes conlleva determinar una serie de actividades y tareas concretas que describen las actuaciones que pueden desarrollar los enfermeros del trabajo en su ámbito laboral.

A su vez dentro de las distintas actuaciones se establece una interrelación con la clasificación taxonómica de diagnósticos de enfermería establecidos por la NANDA Internacional, donde se proponen cada una de las intervenciones de enfermería ${ }^{28,29,30,31}$. 


\section{EJEMPLOS PRÁCTICOS DE PRESCRIPCIÓN ENFERMERA EN EL MEDIO LABORAL ${ }^{35}$}

A continuación se detallan las situaciones más habituales en las que las enfermeras prescriben medicamentos o productos sanitarios. Y se reseñan los diagnósticos más frecuentes que justifican su actuación y las intervenciones enfermeras que se derivan de cada diagnóstico ${ }^{36,37,38,39}$.

La Clasificación de Intervenciones de Enfermería abarca las intervenciones realizadas por todos los profesionales de la Enfermería. Todas las incluidas en la clasificación tienen el propósito de resultar clínicamente útiles aunque algunas sean más generalistas que otras. El uso de la clasificación proporciona las siguientes utilidades:

- Ayuda a mostrar el impacto que tienen los profesionales de la enfermería en el sistema de cuidados de salud.

- Normaliza y define la base de conocimientos de la práctica enfermera.

- Facilita la elección correcta de una intervención de enfermería.

- Permite la comunicación de los tratamientos de enfermería a otros profesionales de la enfermería y a otros miembros del equipo multidisciplinar.

- Permite a los investigadores examinar la eficacia y el coste de los cuidados.

- Facilita la enseñanza en la toma de decisiones clínicas a los profesionales noveles.

- Ayuda a los gestores a planificar de forma más eficaz las necesidades de personal.

- Facilita el desarrollo y uso de sistemas de información entre profesionales enfermeros.

- Permite explicar el contenido de los cuidados al usuario.

Situaciones más habituales:

- Accidentes de trabajo. Politraumatizados.

- Ayuda para dejar de fumar.

- Control de glucemia.

- Diarrea.

- Dietoterapia.

- Estreñimiento.

- Fármacos: administración si precisa.

- Fiebre.

- Hipoglucemia. Manejo de paciente diabético.

- Movilización (en situaciones de accidentes de trabajo).

- Oxigenación. Situaciones de emergencia.

- Primeros auxilios.

- Quemaduras. Roces. Traumas.

- Sutura de heridas.

- Tapones en los oídos.

- Vacunación laboral. 


\begin{tabular}{ll}
\hline & 0038 Riesgo de traumatismo \\
& 0043 Protección inefectiva \\
& 0046 Deterioro de la integridad cutánea \\
Diagnóstico enfermero & 0044 Deterioro de la integridad tisular \\
& 0085 Deterioro de la movilidad física \\
& 0086 Riesgo de disfunción neurovascular periférica \\
& 0145 Riesgo de síndrome postraumático \\
\hline \multirow{2}{*}{ Actuaciones } & Proteger al paciente de lesiones nuevas secundarias al transporte y rescate \\
& evitando el agravamiento \\
\hline \multirow{2}{*}{ Intervenciones enfermeras } & 0910 Inmovilización \\
& 1860 Ayuda con los autocuidados: transferencia \\
& 0840 Cambio de posición \\
& 0844 Cambio de posición: neurológico \\
& 2660 Manejo de la sensibilidad periférica alterada \\
& 6490 Prevención de caídas \\
\hline Tipo de prescripción & Producto sanitario \\
\hline & Elementos de rescate, movilización e inmovilización de accidentados \\
Medicación/Producto & Vendas \\
& Apósitos \\
& Sistemas de almohadillado \\
& Antisépticos \\
\hline
\end{tabular}

\section{Ayuda para dejar de fumar}

084 Conductas generadoras de salud (especificar) 030 Deterioro del intercambio gaseoso 079 Incumplimiento del tratamiento (especificar) 092 Intolerancia a la actividad

Diagnóstico enfermero 031 Limpieza ineficaz de las vías aéreas 078 Manejo inefectivo del régimen terapéutico 099 Mantenimiento inefectivo de la salud 032 Patrón respiratorio ineficaz 241 Perfusión tisular inefectiva cardiopulmonar 094 Riesgo de intolerancia a la actividad

\begin{tabular}{ll}
\hline Actuaciones & Se orienta sobre el tipo de producto que debe combinar con la terapia \\
& para conseguir que el paciente deje de fumar \\
\hline & 4490 Ayuda para dejar de fumar \\
& 3140 Manejo de las vías aéreas \\
& 3250 Mejora de la tos \\
Intervenciones enfermeras & 3321 Terapia de ejercicios: ambulación \\
& 4500 Prevención del consumo de sustancias nocivas \\
& 8180 Consulta por teléfono \\
\hline Tipo de prescripción & Medicación y producto sanitario \\
\hline Medicación/Producto & Terapia sustitutiva de nicotina \\
\hline
\end{tabular}

Control de la glucemia

\begin{tabular}{ll}
\hline Diagnóstico enfermero & 002 Desequilibrio nutricional: por defecto \\
& 001 Desequilibrio nutricional: por exceso \\
\hline \multirow{2}{*}{ Actuaciones } & $\begin{array}{l}\text { La valoración de enfermería ante situaciones de hipoglucemia o hiper- } \\
\text { glucemia permite corregir la situación con aporte de glucosa y disminu- } \\
\text { yendo/suspendiendo temporalmente la dosis de insulina y antidiabéticos } \\
\text { orales, o aumentado su dosis en situación contraria }\end{array}$ \\
\hline \multirow{2}{*}{ Intervenciones enfermeras } & 2130 Manejo de la hipoglucemia \\
& 2120 Manejo de la hiperglucemia \\
\hline Tipo de prescripción & Medicación y producto sanitario \\
\hline \multirow{3}{*}{ Medicación/Producto } & Glucosa/glucagón \\
& Insulina (ajuste de dosis) \\
& Antidiabéticos orales (ajuste de dosis) \\
& Tiras reactivas para determinación de glucemia \\
\hline
\end{tabular}


Diarrea

\begin{tabular}{ll}
\hline Diagnóstico enfermero & $\begin{array}{l}\text { 013 Diarrea } \\
\text { 011 Estreñimiento }\end{array}$ \\
\hline Actuaciones & $\begin{array}{l}\text { Administrar la medicación adecuada o técnicas que ayuden a aliviar sus } \\
\text { efectos secundarios }\end{array}$ \\
\hline & 0460 Manejo de la diarrea \\
& 1100 Manejo de la nutrición \\
& 2390 Prescribir medicación \\
Intervenciones enfermeras & 0410 Cuidados de la piel: tratamiento tópico da incontinencia intestinal \\
& 0440 Entrenamiento intestinal \\
& 0430 Manejo intestinal \\
\hline Tipo de prescripción & Medicación y producto sanitario \\
\hline \multirow{2}{*}{ Medicación/Producto } & Suero oral \\
& Ultralevura (probiótico)
\end{tabular}

\section{Dietoterapia}

084 Conductas generadoras de salud

126 Conocimientos deficientes

102 Déficit de autocuidado: alimentación

027 Déficit de volumen de líquidos

002 Desequilibrio nutricional por defecto

001 Desequilibrio nutricional por exceso

Diagnóstico enfermero

Deterioro de la dentición

013 Diarrea

011 Estreñimiento

093 Fatiga

007 Hipertermia

092 Intolerancia a la actividad

099 Mantenimiento inefectivo de la salud

032 Patrón respiratorio ineficaz

Se evalúa la situación del paciente ajustando la dieta en función de los cambios que se puedan producir durante el tratamiento, adecuándola a la tolerancia o evolución del paciente (diarrea, estreñimiento, gustos, intole-

Actuaciones rancia, dificultad de masticación...). Hay situaciones que pueden obligar a la suspensión de la alimentación y otras en las que hay una prescripción de cambio en el tipo y características de la dieta.

1240 Ayuda para ganar peso

1280 Ayuda para disminuir peso

1710 Mantenimiento de la salud bucal

Intervenciones enfermeras $\quad 1803$ Ayuda en los autocuidados: alimentación

2120 Manejo de la hiperglucemia

2130 Manejo de la hipoglucemia

0460 Manejo de la diarrea

5246 Asesoramiento nutricional

\begin{tabular}{ll}
\hline Tipo de prescripción & Medicamento y producto sanitario \\
\hline Medicación/Producto & Suplementos alimenticios: vitaminas, minerales... \\
\hline
\end{tabular}




\section{Estreñimiento}

\begin{tabular}{ll}
\hline Diagnóstico enfermero & $\begin{array}{l}\text { 011 Estreñimiento } \\
\text { 015 Riesgo de estreñimiento }\end{array}$ \\
\hline \multirow{2}{*}{ Actuaciones } & Aplicar los productos y/o medicamentos adecuados mediante las técnicas \\
& enfermeras según la vía de aplicación del laxante \\
\hline & 0450 Manejo del estreñimiento/impactación \\
& 1100 Manejo de la nutrición \\
& 1400 Manejo del dolor \\
& 2315 Administración de medicación: rectal \\
Intervenciones enfermeras & 4120 Manejo de líquidos \\
& 0430 Manejo intestinal \\
& 0440 Entrenamiento intestinal \\
\hline Tipo de prescripción & Medicación y producto sanitario \\
\hline \multirow{2}{*}{ Medicación/Producto } & Supositorio de glicerina \\
& Micralax \\
\hline
\end{tabular}

\section{Fármacos: administración si precisa}

100 Retraso en la recuperación quirúrgica y/o traumática 011 Estreñimiento

132 Dolor agudo

134 Náuseas

Diagnóstico enfermero 015 Riesgo de estreñimiento

032 Patrón respiratorio ineficaz

035 Riesgo de accidente

041 Respuesta alérgica al látex

047 Riesgo de deterioro de la integridad cutánea

008 Termorregulación ineficaz

\begin{tabular}{|c|c|c|}
\hline Actuaciones & \multicolumn{2}{|c|}{$\begin{array}{l}\text { Ante una orden médica escrita pautada de un fármaco, la enfermería valo- } \\
\text { ra la aplicación de dicho fármaco según sus habilidades y conocimientos }\end{array}$} \\
\hline \multirow{10}{*}{ Intervenciones enfermeras } & \multirow{10}{*}{\multicolumn{2}{|c|}{$\begin{array}{l}2210 \text { Administración de analgésicos } \\
2840 \text { Administración de anestesia } \\
2300 \text { Administración de medicación } \\
2313 \text { Administración de medicación: intramuscular } \\
2314 \text { Administración de medicación: intravenosa } \\
2310 \text { Administración de medicación: oftálmica } \\
2304 \text { Administración de medicación: oral } \\
2308 \text { Administración de medicación: ótica } \\
2315 \text { Administración de medicación: rectal } \\
2316 \text { Administración de medicación: tópica }\end{array}$}} \\
\hline & & \\
\hline & & \\
\hline & & \\
\hline & & \\
\hline & & \\
\hline & & \\
\hline & & \\
\hline & & \\
\hline & & \\
\hline Tipo de prescripción & \multicolumn{2}{|l|}{ Medicación } \\
\hline \multirow{8}{*}{ Medicación/Producto } & Analgésicos & Broncodilatadores \\
\hline & Ansiolíticos & Anticonvulsivantes \\
\hline & Sedantes & Antidiarreicos \\
\hline & Hipnóticos & Laxantes \\
\hline & Protectores gástricos & Insulina \\
\hline & Antiácidos & Glucosa \\
\hline & Antitérmicos & Oxigenoterapia \\
\hline & \multicolumn{2}{|l|}{ Antiinflamatorios } \\
\hline
\end{tabular}




\section{Fiebre}

\begin{tabular}{ll}
\hline Diagnóstico enfermero & $\begin{array}{l}\text { 007 Hipertermia } \\
005 \text { Riesgo de desequilibrio de la temperatura corporal }\end{array}$ \\
\hline \multirow{2}{*}{ Actuaciones } & $\begin{array}{l}\text { La enfermería evalúa, analiza la situación, prescribe y explica la pauta de } \\
\text { administración de fármacos. A su vez administra la medicación adecuada } \\
\text { o aplica técnicas que ayudan a conseguir la normalización de la tempera- } \\
\text { tura. }\end{array}$ \\
\hline 1100 Manejo de nutrición \\
1380 Aplicación de calor o frío \\
2390 Prescribir medicación \\
3320 Oxigenoterapia \\
3780 Tratamiento de exposición al calor \\
3900 Regulación de la temperatura \\
\hline Tipo de prescripción & Medicación \\
\hline Medicación/Producto & Antitérmicos \\
\hline
\end{tabular}

\section{Hipoglucemia. Manejo del paciente diabético}

\begin{tabular}{|c|c|}
\hline Diagnóstico enfermero & $\begin{array}{l}002 \text { Desequilibrio nutricional: por defecto } \\
001 \text { Desequilibrio nutricional: por exceso }\end{array}$ \\
\hline Actuaciones & $\begin{array}{l}\text { La hiploglucemia causa pérdida de conciencia en ocasiones que supo- } \\
\text { ne también un compromiso respiratorio por caída de la lengua sobre la } \\
\text { hipofaringe. La puesta en marcha del algoritmo del soporte vital básico, } \\
\text { acompañado de la administración de glucosa hipertónica intravenosa hace } \\
\text { que revierta el cuadro en un alto porcentaje de casos. } \\
\text { El glucagón, la insulina, etc. son fármacos que el paciente y/o su familia } \\
\text { manejan de forma habitual debido a descompensaciones }\end{array}$ \\
\hline Intervenciones enfermeras & $\begin{array}{l}2130 \text { Manejo de la hipoglucemia } \\
2120 \text { Manejo de la hiperglucemia }\end{array}$ \\
\hline Tipo de prescripción & Medicación y producto sanitario \\
\hline Medicación/Producto & $\begin{array}{l}\text { Glucosa/glucagón } \\
\text { Insulina (ajuste de dosis) } \\
\text { Antidiabéticos orales (ajuste de dosis) } \\
\text { Tiras reactivas para determinación de glucemia }\end{array}$ \\
\hline
\end{tabular}


Movilización (en situaciones de accidente de trabajo)

\begin{tabular}{|c|c|}
\hline Diagnóstico enfermero & $\begin{array}{l}038 \text { Riesgo de traumatismo } \\
149 \text { Riesgo de síndrome de estrés del traslado } \\
035 \text { Riesgo de lesión } \\
094 \text { Riesgo de intolerancia a la actividad } \\
155 \text { Riesgo de caídas } \\
092 \text { Intolerancia a la actividad } \\
093 \text { Fatiga } \\
085 \text { Deterioro de la movilidad física } \\
090 \text { Deterioro de la habilidad para la traslación } \\
088 \text { Deterioro de la deambulación }\end{array}$ \\
\hline Actuaciones & Ayuda y colaboración en el traslado con una movilización adecuada \\
\hline Intervenciones enfermeras & $\begin{array}{l}1400 \text { Manejo del dolor } \\
2300 \text { Administración de medicación } \\
2390 \text { Prescribir medicación } \\
3140 \text { Manejo de vías aéreas } \\
3320 \text { Oxigenoterapia } \\
3390 \text { Ayuda en la ventilación } \\
4010 \text { Precauciones en las hemorragias } \\
4020 \text { Disminución de la hemorragia } \\
4190 \text { Canalización venosa periférica } \\
4250 \text { Manejo del shock } \\
6420 \text { Restricción de zonas de movimiento } \\
6490 \text { Prevención de caídas } \\
0910 \text { Inmovilización }\end{array}$ \\
\hline Tipo de prescripción & Productos sanitarios \\
\hline Medicación/Producto & Dispositivos para el cambio de postura y traslado \\
\hline
\end{tabular}

\section{Oxigenación. Situaciones de emergencia}

\begin{tabular}{ll}
\hline & 033 Deterioro de la respiración espontánea \\
Diagnóstico enfermero & 092 Intolerancia a la actividad \\
& 032 Patrón respiratorio ineficaz \\
\hline & Se valora la necesidad o conveniencia de administrar oxigenoterapia o \\
cambiar la modalidad, concentración, flujo, etc. & En base a una valoración enfermera o decisión terepéutica médica habrá \\
una administración de nebulizadores de suero salino u otros fluidos y/o & sustancias farmacológicas que ayudan a la hidratación y permeabilización \\
de la vía aérea. Siempre y cuando no haya contraindicación específica de & las mismas, alergias o posibles dudas. \\
Actuaciones & 3320 Oxigenoterapia \\
& 3230 Fisioterapia respiratoria \\
& 2311 Administración de medicación: inhalación \\
Intervenciones enfermeras & Medicación y productos sanitarios \\
\hline Tipo de prescripción & Oxigenoterapia con mascarilla y gafas nasales \\
& Balón autohinchable (ambú) \\
Inhaladores & Nebulizadores \\
\hline \multirow{2}{*}{ Medicación/Producto } &
\end{tabular}




\section{Primeros auxilios}

\begin{tabular}{ll}
\hline Diagnóstico enfermero & \\
\hline & Cuidados al paciente con necesidades de oxigenación \\
& Cuidados y tratamiento específicos de heridas y curas \\
& Vacunación antitetánica \\
& Tratamiento de los vómitos \\
& Mejorar la TA \\
& Disponer de vías de acceso venoso \\
& HTA súbita \\
& Actuaciones ante la presencia de hipoglucemia, caídas... \\
Actuaciones & Aspiración de secreciones bronquiales \\
& Evitar sangrado activo suspendiendo medicación anticoagulante y aplicando \\
& hemostáticos \\
\hline Intervenciones enfermeras & 3320 Oxigenoterapia \\
& 6530 Manejo de la inmunización/vacunación \\
& 3620 Sutura \\
& 2840 Administración de anestesia \\
& 4200 Terapia intravenosa \\
\hline Tipo de prescripción & Medicación y productos sanitarios \\
\hline & Oxígeno \\
& Anestésicos \\
Vacuna antitetánica \\
Materiales de cura y sutura \\
Antieméticos \\
Sueroterapia \\
Antihipertensivos \\
Tiras reactivas de glucemia \\
Glucosa \\
Fármacos hemostáticos (spongostam) \\
\end{tabular}

\section{Quemaduras. Roces. Traumas}

\begin{tabular}{ll}
\hline Diagnóstico enfermero & \\
\hline \multirow{2}{*}{ Actuaciones } & $\begin{array}{l}\text { Curas de heridas postquirúrgicas, úlceras vasculares, pie diabético, } \\
\text { abrasiones, etc. }\end{array}$ \\
\hline & 3660 Cuidado de las heridas \\
& 3620 Sutura \\
& 2840 Administración de anestesia \\
Intervenciones enfermeras & 3440 Cuidados del sitio de incisión \\
& 4062 Cuidados circulatorios: insuficiencia arterial \\
& 4066 Cuidados circulatorios: insuficiencia venosa \\
\hline Tipo de prescripción & Medicación y productos sanitarios \\
\hline & Suero fisiológico \\
& Antisépticos: Betadine ${ }^{\circledR}$, Clorhexidina ${ }^{\circledR}$ \\
& Cicatrizantes: Linitul ${ }^{\circledR}$, Tulgraso \\
& Gasas, vendas, esparadrapo \\
& Gasa para taponamiento nasal \\
& Anestésico local \\
& Suturas \\
Sistema de drenaje \\
Desbridantes
\end{tabular}




\section{Sutura de heridas}

100 Retraso en la recuperación quirúrgica

004 Riesgo de infección

Diagnóstico enfermero 044 Deterioro de la integridad tisular

046 Deterioro de la integridad cutánea

047 Riesgo de deterioro de la integridad cutánea

\begin{tabular}{ll}
\hline Actuaciones & $\begin{array}{l}\text { Procurar una disminución del dolor y aumentando el confort ante la sutura. } \\
\text { Administrar anestésico localmente. }\end{array}$ \\
\hline Intervenciones enfermeras & $\begin{array}{l}\text { 3620 Sutura } \\
\text { 3660 Cuidado de las heridas }\end{array}$ \\
\hline Tipo de prescripción & Medicación y producto sanitario \\
\hline Medicación/Producto & $\begin{array}{l}\text { Anestesia local } \\
\text { Antisépticos } \\
\text { Seda (material de sutura) }\end{array}$ \\
\hline
\end{tabular}

Tapones en los oídos

\begin{tabular}{ll}
\hline Diagnóstico enfermero & $\begin{array}{l}\text { 0108 Déficit de autocuidado: baño/higiene } \\
1221 \text { Trastorno de la percepción sensorial: auditiva }\end{array}$ \\
\hline Actuaciones & $\begin{array}{l}\text { En la consulta de enfermería se dispone y prepara el conducto auditivo } \\
\text { con los productos y medicación (se suelen utilizar y recomendar sin receta } \\
\text { médica) adecuados para facilitar la extracción del cerumen. }\end{array}$ \\
\hline Intervenciones enfermeras & $\begin{array}{l}\text { 1640 Cuidados de los oídos } \\
\text { 4974 Mejorar comunicación: déficit auditivo }\end{array}$ \\
\hline Tipo de prescripción & Medicamento \\
\hline Medicación/Producto & $\begin{array}{l}\text { Otocerum } \\
\text { Agua oxigenada } \\
\text { Taponoto }\end{array}$ \\
\hline
\end{tabular}

Vacunación laboral

077 Afrontamiento inefectivo de la comunidad

084 Conductas generadoras de salud (especificar)

Diagnóstico enfermero

126 Conocimientos deficientes (especificar)

076 Disposición para mejorar el afrontamiento de la comunidad

081 Manejo inefectivo del régimen terapéutico de la comunidad

004 Riesgo de infección

\begin{tabular}{ll}
\hline & Según los programas o protocolos la enfermera decide administrar la \\
vacuna si las condiciones son las adecuadas. \\
Vigila la postpunción y recomienda medicamentos o terapias si aparecen \\
complicaciones
\end{tabular}


Medicamentos y productos sanitarios administrados más frecuentes ${ }^{40}$

\begin{tabular}{ll}
\hline Analgésicos & Solución antiséptica \\
Apósitos & Suplementos dietéticos \\
Antiinflamatorios & Aerosoles respiratorios \\
Antibióticos & Colirios \\
Vacunas & Suplementos vitamínicos \\
Insulina & Antigripales \\
Antitérmicos & Vendajes \\
Sueros & Antidiarreicos \\
Protectores gástricos & Ablandadores de cerumen \\
Laxantes & Colutorios \\
Anestésicos & Sustitutivos del tabaco \\
Desbridantes & \\
\hline
\end{tabular}

CONCLUSIONES

La especialidad de Enfermería del Trabajo la constituyen unos profesionales cualificados cuya titulación les faculta para proporcionar los cuidados propios de su disciplina $^{44}$.

La Enfermería del Trabajo tiene asignadas competencias específicas dentro de distintas áreas en el marco de los servicios de prevención de riesgos laborales: preventiva, asistencial, legal y pericial, gestión, y docencia e investigación ${ }^{45}$.

Un lenguaje común propio de la profesión enfermera permite de una parte una comunicación rápida y universal, y de otra la sistematización del trabajo enfermero ${ }^{46}$. La integración de los diagnósticos e intervenciones de Enfermería en la práctica diaria deben constituir uno de los objetivos profesionales prioritarios.

Los centros de trabajo constituyen espacios inmejorables para promover intervenciones preventivas dirigidas tanto al conjunto de la población laboral como a determinados colectivos profesionales según sus riesgos específicos. De esta manera todo programa de salud pública desarrollado en el entorno laboral puede extender sus efectos beneficiosos a la población general. Por ello resultan interesantes y factibles cuantas iniciativas tengan como objetivo final la prestación de unos cuidados enfermeros estructurados y de calidad en el ámbito de nuestras competencias como especialistas en Enfermería del Trabajo. Y todo ello siendo conscientes de las dificultades y problemas que conllevan estas actuaciones.

Dentro de los servicios de prevención de riesgos laborales el abordaje de la prescripción enfermera se tiene que contemplar desde la autonomía y responsabilidad profesional propia y la existencia de un equipo multidisciplinar.

La prescripción enfermera tiene que enmarcarse en las competencias que aseguren una indicación efectiva, eficaz y segura mediante programas de formación específicos.

La Enfermería del Trabajo garantiza una buena práctica profesional y la seguridad de los pacientes si la práctica está normalizada, si se definen los estándares de calidad y el lenguaje es homogéneo en todas sus intervenciones ${ }^{47}$.

La optimización de los recursos es fundamental en toda atención sanitaria. Una herramienta que contribuye a la consecución de este objetivo son las intervenciones de Enfermería, validadas con una metodología científica de las cuales se conocen el coste material, personal y de tiempo empleado en su ejecución. Su aplicación contribuye a la homogeneización del lenguaje, la sistematización de los procedimientos y la rentabilización del trabajo ${ }^{48}$. 
La capacidad de la enfermería en el marco de la prestación de cuidados enfermeros de seleccionar guiados por el criterio profesional diferentes materiales, productos sanitarios y medicación encaminados a satisfacer las necesidades de salud del usuario y la población apoyados por el juicio clínico enfermero ha de constituir uno de los ejes primordiales de la actuación enfermera.

\section{BIBLIOGRAFÍA}

1. Johnson, M. et cols. Interrelaciones NANDA, NOC y NIC. Diagnósticos enfermeros, resultados e intervenciones. Elsevier Mosby. 2006.

2. Maas, ML. et cols. Classification of nursing interventions. Journal of Professional Nursing, 6(3), 151-157. Explica cómo debe ser el desarrollo de un lenguaje normalizado en las intervenciones de enfermería. El artículo se divide en cuatro partes: introducción, razones de un sistema de clasificación, análisis de los sistemas existentes y una descripción de los primeros trabajos realizados por el equipo de investigación del Iowa Project Interventions para el desarrollo de la Clasificación de Intervenciones de Enfermería. 1990.

3. Maas, ML. et cols. Classification of nursing interventions. Journal of Professional Nursing, 6 (3), 151-157. Explica cómo debe ser el desarrollo de un lenguaje normalizado en las intervenciones de enfermería. El artículo se divide en cuatro partes: introducción, razones de un sistema de clasificación, análisis de los sistemas existentes y una descripción de los primeros trabajos realizados por el equipo de investigación del Iowa Project Interventions para el desarrollo de la Clasificación de Intervenciones de Enfermería. 1990.

4. Zielstroff, R.D. Nursing Information and Data Set Evaluation Center Committee. NIDSEC Standards and Scoring Guidelines (monograph). American Nurse Association. Pub. N Q-2. 1997.

5. Zielstroff, R.D, Teich, J.M, Paterno, M.D, Segal, M, Kuperman, G.J, Hiltz, F.L. P-CAP: A high-level tool for entering and processing clinical practice guidelines. CG Chute (ed). Proceedings, 1998 AMIA Annual Fall Symposium (JAMIA Symposium Supplement) Fall 1998, p. 478-82.

6. McCloskey, J.C. y Bulechek, G.M. Clasificación de Intervenciones de Enfermería: implicación de los diagnósticos de enfermería. Carroll-Johnson y M. Paquette (Eds.). Clasificación de Diagnósticos de Enfermería: Procedimientos de la Décima Conferencia (307-310). Philadelphia: J.B. Lippincott. 1994. Presenta el trabajo realizado por el equipo de investigación del Proyecto de Intervenciones de Iowa hasta 1993 en el desarrollo de la Clasificación de Intervenciones de Enfermería. El propósito global del trabajo de clasificación fue elaborar y validar una taxonomía de Intervenciones de Enfermería que aplica en los diagnósticos enfermeros. Además analiza asuntos clave relacionados con los propios diagnósticos.

7. Moss, L. y Donahue, W. Presentación de la Clasificación de Intervenciones de Enfermería. New York: National League for Nursing. 1994. Análisis de la Clasificación de Intervenciones de Enfermería (CIE) que incluye entrevistas con los miembros y enfermeros/as del equipo de investigación del CIE en cinco campos de prueba donde se implementaron las intervenciones. Constituyen una buena orientación tanto para el personal clínico como para los estudiantes de Enfermería.

8. Aquilino, M.L. y Keenan, G. Having our say: Nursing's standarized nomenclatures. American Journal of Nursing, 2000. 100(7), 33-38.

9. Dochterman, J. y Jones, D. (Eds). Unifying nursing languages: The harmonization of NANDA, NIC, and NOC. Washington, DC: American Nurses Association. 2003. Trata la necesidad de una taxonomía de intervenciones de enfermería que incluya todos los tratamientos de enfermería realizados por las enfermeras con sus pacientes.

10. McCloskey J, Bulechek GM. Clasificación de intervenciones de enfermería. Nursing Interventions Classification (NIC). 3. ${ }^{a}$ edición. Harcourt. 2003.

11. NANDA International 2005. Nursing diagnoses: definitions \& classification 2005-2006. Philadelphia. 277.

12. Dochterman J.M \& Bulechek G.M. (Eds). 2004. Nursing interventions classification (NIC). 4th ed. St. Louis: Mosby.

13. Moorhead S, Johnson M. y Maas M. (Eds). 2004. Nursing outcomes classification (NOC). $3^{\text {rd }}$. St. Louis: Mosby.

14. Consejo General de Enfermería. Marco referencial para la prescripción enfermera. 2006.

15. Boletín Oficial del Estado. Ley 29/2006 de 26 de julio de garantías y uso racional de los medicamentos y productos sanitarios. BOE núm 178 de 27/07/2006 [Acceso 9 de diciembre de 2010]. Disponible en: http:// www.boe.es/boe/dias/2006/07/27/pdfs/A28122-28165.pdf

16. Autoridad de los profesionales de enfermería para prescribir: ¿un camino a seguir? Editorial Nursing 2002, volumen 20 , núm. 2 .

17. Rubio C, Cuesta A. La capacidad de prescribir de las enfermeras. Enfermería Integral. 1999, 50: XXXV-XLII.

18. Bigbee, G. Territoriality and prescriptive authority for nurse practitioners. Nurse Health Care. 1984. 5 (2): 106-110. 
19. Boletín Oficial del Estado. Ley 44/2003, de 21 de noviembre, de Ordenación de las profesiones sanitarias. BOE núm 280 de 22/11/2003. [Acceso 21 de abril de 2010]. Disponible en: http://www.boe.es/boe/ dias/2003/11/22/pdfs/A41442-41458.pdf

20. Boletín Oficial del Estado. Real Decreto 39/1997, de 17 de enero, por el que se aprueba el Reglamento de los Servicios de Prevención. BOE núm 27 de 31/1/1997. [Acceso 25 de abril de 2010]. Disponible en: http:// www.boe.es/boe/dias/1997/01/31/pdfs/A03031-03045.pdf

21. Las competencias profesionales de la enfermería del trabajo. Sociedad Catalana de Seguridad y Medicina de Trabajo. Grupo de Enfermería del Trabajo.

22. Boletín Oficial del Estado. Ley 44/2003, de 21 de noviembre, de Ordenación de las profesiones sanitarias. BOE núm 280 de 22/11/2003. [Acceso 21 de abril de 2010]. Disponible en: http://www.boe.es/boe/ dias/2003/11/22/pdfs/A41442-41458.pdf

23. Boletín Oficial del Estado. Real Decreto 450/2005, de 22 de abril, sobre especialidades de Enfermería. BOE núm 108 de 6/5/2005. [Acceso 21 de abril de 2010]. Disponible en: http://www.boe.es/boe/dias/2005/05/06/ pdfs/A15480-15486.pdf

24. Boletín Oficial del Estado. Real Decreto 450/2005, de 22 de abril, sobre especialidades de Enfermería. BOE núm 108 de 6/5/2005. [Acceso 21 de abril de 2010]. Disponible en: http://www.boe.es/boe/dias/2005/05/06/ pdfs/A15480-15486.pdf

25. Vázquez J.A. Situación de la enfermería del trabajo en formación pregrado en las distintas universidades públicas españolas tras la aprobación de la especialidad en el Real Decreto 450/2005.

26. Las competencias profesionales de la enfermería del trabajo. Sociedad Catalana de Seguridad y Medicina del Trabajo. Grupo de Enfermería del Trabajo.

27. Benito J. Cartera de Servicios de la Enfermería del Trabajo.

28. Diagnósticos enfermeros: definición y clasificación 2005-2006. NANDA International. North American Nursing Diagnosis Association. Elsevier. 2005.

29. Johnson M, Maas M, Moorhead S. Clasificación de resultados de enfermería (NOC). 2. ${ }^{\text {a }}$ edición. Harcourt.

30. McCloskey J y Bulechek GM. Clasificación de intervenciones de enfermería (NIC). 3. ${ }^{a}$ edición. Harcourt

31. Gordon M. Manual de diagnósticos enfermeros. 10. ${ }^{a}$ edición. Harcourt.

32. Revisión de la Enfermería de Salud Laboral en el marco de la Ley 31/1995 de Prevención de Riesgos Laborales. Rev. SEMST 2004; 0: 28-32.

33. Las competencias profesionales de la enfermería del trabajo. Sociedad Catalana de Seguridad y Medicina del Trabajo. Grupo de Enfermería del Trabajo.

34. Benito, J. Cartera de Servicios de la Enfermería del Trabajo.

35. Listado de medicamentos y productos sanitarios prescritos por las enfermeras en España. Consejo General de Enfermería. 2007.

36. Diagnósticos enfermeros: definición y clasificación 2001-2002. NANDA. Harcourt.

37. Johnson M, Maas M, Moorhead S. Clasificación de resultados de enfermería (NOC). 2. ${ }^{\text {a }}$ edición. Harcourt.

38. McCloskey J, Bulechek GM. Clasificación de intervenciones de enfermería (NIC). 3. ${ }^{a}$ edición. Harcourt. 2003

39. Gordon, M. Manual de diagnósticos enfermeros. 10. ${ }^{a}$ edición. Harcourt.

40. Listado de medicamentos y productos sanitarios prescritos por las enfermeras en España. Consejo General de la Enfermería. 2007.

41. Santos M, et al. Gestión compartida de la demanda asistencial entre médicos y enfermeras en atención primaria. Enferm Comun. 2005;1(1):35-42

42. Naegle MA. Prescription drug regulation: implications for nursing and health care delivery response of the American Nurses Association. Impact of prescription drug diversion control system on medical practice and patient care. Unitet States Department of Health and Human Services. 1993; 228-34.

43. Autoridad de los profesionales de enfermería para prescribir: ¿un camino a seguir? Editorial Nursing 2002, volumen 20, núm 2.

44. Decálogo profesional de la enfermería española. Consejo General de la Enfermería.

45. Decálogo profesional de la enfermería española. Consejo General de la Enfermería.

46. McCloskey, J.C. y Bulechek, G.M. Normalización del lenguaje para tratamientos de enfermería: un análisis de las cuestiones. Nursing Outlook, 42(2) 5-63.

47. Decálogo profesional de la enfermería española. Consejo General de la Enfermería.

48. McCloskey J.C, Bulechek G.M. Clasificación de Intervenciones de Enfermería. Grupo de revisión del Consejo General de la Enfermería de la edición española de la Clasificación de Intervenciones de Enfermería. 1994

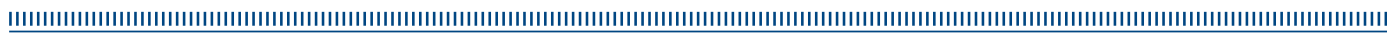

